\title{
PATTERN AND PREDICTORS OF DEATH FROM ALUMINUM AND ZINC PHOSPHIDE POISONING: A TWO YEARS PROSPECTIVE STUDY
}

\author{
Mofrih Mohamed Hegazy ${ }^{*}$, Situhom Elsayed Elagamy ${ }^{*}$, Eman Abdel-Azeem Salem ${ }^{* *}$ \\ *Forensic Medicine and Clinical Toxicology department, Faculty of Medicine, Menoufia University \\ **Public Health and Community Medicine department, Faculty of Medicine, Menoufia University \\ Corresponding author: \\ Mofrih Mohamed Hegazy \\ Email: mofrihhegazy@yahoo.com
}

\begin{abstract}
Background: Aluminum phosphide (ALP) and zinc phosphide pesticides are widely used as grain preservatives in Egypt. It was associated with high morbidity and mortality rates. Aim: To investigate the pattern and predictors of mortality related to aluminum and zinc phosphide poisoning. Methods: A two years prospective study was conducted on 399 patients with acute aluminum and zinc phosphide poisoning (124 non-survivors and 275 survivors) who were admitted to Menoufia University Poison and Dependence Control center (MPCC), during the period from 1st of July 2016 to the end of June 2018. For each patient, demographic and poisons data were taken. Also, clinical examination, laboratory investigations, ECG and Echocardiography were performed. Results: Phosphide poisoning predominated among cases aged $<18$ years $(46.8 \%)$, females $(68.7 \%)$, students $(48.9 \%)$ who attempted suicide $(92.2 \%)$. The majority of non-survivors took aluminum phosphide tablets (97.6\%) while most of survivors took zinc phosphide powder $(78.5 \%)$. There was a significant difference as regards poisoning severity score (PSS), severe PSS among nonsurvivors (91.9\%) while mild PSS among survivors (77.4\%). Severe left ventricular dysfunction was the most predicting factor for mortality in the studied cases $(\mathrm{OR}=9.35$; $\mathrm{CI}$ : 1.156-5.26, $\mathrm{P}<0.001)$ followed by higher dose, need for mechanical ventilation, more than 2 hours' time delay, acidosis and hypotension. Conclusion: The mortality from aluminum and zinc phosphide poisoning can be predicted by intake one tablet or more of AlP with delayed time from intake to seek medical care, left ventricular dysfunction, hypotension and acidosis. These finding necessitate the need for public health policies to restrict the accessibility and use of phosphides in Egypt with the awareness of the public to the high fatality of these poisons.
\end{abstract}

Keywords: Pattern; Predictors; Deaths; Aluminum phosphide (ALP); Zinc phosphide.

\section{INTRODUCTION}

Aluminum and zinc phosphides pesticides are used worldwide to prevent the harmful effects of pests and rodents on stored grains. Phosphine gas was released when aluminum phosphide contact with water or hydrochloric acid in the stomach. The toxic effects of phosphine was due inhibition of cytochrome oxidase system in the mitochondria and consequent impairment of tissue respiration (Valmas et al., 2008). In addition, phosphine had a fatal direct toxic effects on cardiac muscles and adrenal glands leading to a circulatory collapse and may lead to death (Shah et al., 2009). 
Aluminum phosphide (AlP) poisoning mortality rates vary from $40 \%$ to $80 \%$ worldwide (Chaudhry \& Rai, 2014). The identified fatal dose of AlP is $0.15-0.5 \mathrm{~g}$. The time elapsed from ingestion of AlP to death ranged from about from 1 to 48 hours, most of the patients die within the first 24 hours from poison intake. The most common cause of death as reported by previous studies was cardiac dysrhythmia (Wahab et al., 2009). Also, the mortality rate of zinc phosphide poisoning is around 37-100\% (Sogut, 2011).

Aluminum and Zinc phosphide, effective grain preservatives are frequently used in silos of Menoufia governorate. However, exposure to these cheap and easily accessible chemicals has provoked poisoning with a high fatality rate. Therefore, this study was conducted to identify the pattern of aluminum and zinc phosphide poisoning and investigate their predictive factors of mortality.

\section{SUBJECTS AND METHODS}

This was a prospective study conducted on all cases of aluminum and zinc phosphide poisoning who were admitted to Menoufia University Poison and Dependence Control Center (MPCC), Egypt during the period from the 1st of July 2016 to the end of June 2018. MPCC is the only referral center for the cases of poisoning in Menoufia governorate.

A total of 399 cases of phosphide (180 aluminum phosphide and 219 zinc phosphide poisoned cases) poisoning were recruited in the present study. Data was obtained from the patient or his relative about; socio-demographic data (including age, sex, residence, marital status, occupation and smoking habits ), intoxication data ( type of phosphide poison, manner of intake, dose, formula, time elapsed since intake), data regarding clinical assessment; clinical poison severity score according to the method described by Persson et al. (1998). Investigations like measurement of arterial blood gases, blood electrolytes, $\mathrm{pH}$, liver and kidney function tests were performed. Silver nitrate test in gastric content were analyzed for all the studied cases (Ahmed, 2017). Serum cholinesterase levels were assessed to all the studied cases (Waber, 1966). In cases with positive silver nitrate test and decreased serum cholinesterase levels, thin layer chromatography was performed for exclusion of mixed toxicity with organophosphate or carbamate (Kaplan et al.,1989).

ECG and Echocardiography were performed and left ventricular function was assessed by ejection fraction (Yancy et al., 2013). In addition, the outcome of all cases was reported.

Ethical considerations: The study was approved by Menoufia University ethical committee. Informed valid consent was taken from each patient or their relatives prior to the beginning of the study. To insure confidentiality, samples were coded and anonymously stored.

Statistical analysis: The statistical analysis was performed by using IBM personal computer and statistical package SPSS version 22. The description of the data was done in the form of percentage (\%) for qualitative data and mean and standard deviation (SD) for qualitative data. For comparison between survivors and non-survivor groups, student $\mathrm{t}$ - test was used for quantitative variables and the Chi-square $\left(\chi^{2}\right)$ test was used for categorical variables. Binary logistic regression analysis was performed to test the independent effects of the predictors of death from aluminum and zinc phosphides poisoning. $\mathrm{P}$ value $<0.05$ was considered statistically significant. 


\section{RESULTS}

Three hundred ninety nine patients had aluminum (180 cases) and zinc (219 cases) phosphide poisoning. The commonest age of the studied cases was less than 18 (46.9\%) and from 18-30 $(33.6 \%)$ years. Two hundred seventy four patients $(68.7 \%)$ were female and $125(31.3 \%)$ were male. Most of cases were from rural area $(65.9 \%)$. About half of the cases were students $(48.9 \%)$ and about two thirds of the series were single $(67.2 \%)$. The need for admission to ICU and mechanical ventilation (respiratory rate $>35 / \mathrm{min}, \mathrm{PaO}_{2}<50 \mathrm{mmHg}$ at home air, $\mathrm{PaCO}_{2}>60 \mathrm{mmHg}$, blood pressure < 80/50, severe metabolic acidosis and patient isn't respond to supportive to treatment) were significantly more among non-survivors than survivors $(\mathrm{P}<0.001)$ as shown in Table 1.

There was a significant difference between survivors and non-survivors as regards the poison type $(\mathrm{P}<0.001)$. The majority of non-survivors took aluminum phosphide tablets $(97.6 \%)$ while most of survivors took zinc phosphide powder (78.5\%). Suicide attempts were the predominant manner in all cases $(92.2 \%)$ while accidental intake presented in only (7.8\%) of them. Oral intake was the route of intake in all cases. Tablets in a dose of more than one were significantly predominant among non-survivors than survivors while zinc phosphide powder in a dose of less than half packet was significantly predominant among survivors than non-survivors. The referred cases were significantly more in non-survivors than survivors $(\mathrm{P}<0.001)$. Time delay between poison ingestion and medical care of $>2$ hours was significantly more predominant in nonsurvivors than survivors $(\mathrm{P}<0.001)$ as shown in Table 2.

As regards clinical manifestations in Table 3, the presentation with consciousness changes, hypothermia, bradycardia, hypotension, tachypnea, cyanosis, abdominal pain, vomiting and severe poisoning severity score was significantly more frequent in nonsurvivors than survivors $(\mathrm{P}<0.001)$.

Investigations of the studied patients were illustrated in Table 4. It showed that, elevated serum creatinine, hypokalemia, hyperkalemia, hypomagnesaemia, metabolic acidosis and hypoxemia were significantly more prevalent among the non-survivors than survivors. Moreover, moderate to severe left ventricular dysfunction as assessed by Echocardiography were more presented in the non-survivors than survivors $(\mathrm{P}<0.001)$.

Based on binary logistic regression analysis for relevant predictors of mortality in aluminum and zinc phosphide poisoning cases, severe left ventricular dysfunction was the most predicting factor for mortality in the studied cases $(\mathrm{OR}=9.35$; CI: 1.156-5.26, $\mathrm{P}<0.001)$ followed by aluminum phosphide dose of $\geq 1.7$ gram $\quad(\mathrm{OR}=$ 7.65 and 7.30; respectively). Moreover, need for mechanical ventilation (for hypoxemia or severe metabolic acidosis or hypotension not responding to supportive treatment), more than 2 hours' time delay, metabolic acidosis and hypotension were significant predictors of death in phosphide poisoning cases $(\mathrm{OR}=5.36,2.65,1.86$ and 1.46; respectively) as shown in Table 5 . 
Table 1: Characteristics of aluminum and zinc phosphide poisoning cases as regards their outcome

\begin{tabular}{|c|c|c|c|c|c|}
\hline Characteristics & $\begin{array}{c}\text { Non- } \\
\text { survivors } \\
(\mathrm{n}=124) \\
\text { No }(\%)\end{array}$ & $\begin{array}{c}\text { Survivors } \\
(\mathrm{n}=275) \\
\text { No }(\%)\end{array}$ & $\begin{array}{c}\text { Total } \\
(\mathrm{n}=399) \\
\text { No }(\%)\end{array}$ & $\chi^{2}$ & p-value \\
\hline $\begin{array}{l}\text { Age (years): } \\
<18 \\
18-30 \\
30-40 \\
>40\end{array}$ & $\begin{array}{c}65(52.4) \\
42(33.9) \\
10(8.1) \\
7(5.6)\end{array}$ & $\begin{array}{c}122(44.4) \\
92(33.5) \\
42(15.2) \\
19(6.9)\end{array}$ & $\begin{array}{c}187(46.9) \\
134(33.6) \\
52(13.0) \\
26(6.5)\end{array}$ & 4.8 & 0.187 \\
\hline $\begin{array}{l}\text { Sex: } \\
\text { Males } \\
\text { Females }\end{array}$ & $\begin{array}{l}31(25.0) \\
93(75)\end{array}$ & $\begin{array}{c}94(34.2) \\
181(65.8)\end{array}$ & $\begin{array}{l}125(31.3) \\
274(68.7)\end{array}$ & 3.35 & 0.067 \\
\hline $\begin{array}{l}\text { Residence: } \\
\text { Rural } \\
\text { urban }\end{array}$ & $\begin{array}{l}90(72.6) \\
34(27.4)\end{array}$ & $\begin{array}{l}173(62.9) \\
102(37.1)\end{array}$ & $\begin{array}{l}263(65.9) \\
136(34.1)\end{array}$ & 3.56 & 0.059 \\
\hline $\begin{array}{l}\text { Marital Status: } \\
\text { Single } \\
\text { married }\end{array}$ & $\begin{array}{l}89(71.8) \\
35(28.2)\end{array}$ & $\begin{array}{l}179(65.1) \\
96(34.9)\end{array}$ & $\begin{array}{l}268(67.2) \\
131(32.8)\end{array}$ & 1.73 & 0.188 \\
\hline $\begin{array}{l}\text { Occupation: } \\
\text { Employers } \\
\text { Farmers \&workers } \\
\text { House wife } \\
\text { Students } \\
\text { Not working }\end{array}$ & $\begin{array}{c}9(7.3) \\
10(8.1) \\
30(24.2) \\
68(54.8) \\
7(5.6)\end{array}$ & $\begin{array}{c}14(5.2) \\
51(18.5) \\
65(23.6) \\
127(46.2) \\
18(6.5)\end{array}$ & $\begin{array}{c}23(5.8) \\
61(15.3) \\
95(23.8) \\
195(48.9) \\
25(6.2)\end{array}$ & 8.27 & 0.082 \\
\hline $\begin{array}{l}\text { Smoking habits: } \\
\text { Smokers } \\
\text { Non-smokers }\end{array}$ & $\begin{array}{c}8(6.5) \\
116(93.5)\end{array}$ & $\begin{array}{c}34(12.4) \\
241(87.6)\end{array}$ & $\begin{array}{c}42(10.5) \\
357(89.5)\end{array}$ & 3.17 & 0.075 \\
\hline $\begin{array}{l}\text { Admission to ICU: } \\
\text { Yes } \\
\text { No }\end{array}$ & $\begin{array}{c}108(87.1) \\
16(12.9)\end{array}$ & $\begin{array}{l}149(54.2) \\
126(45.8)\end{array}$ & $\begin{array}{c}257(89.5) \\
42(10.5)\end{array}$ & 40.39 & $<0.001$ \\
\hline $\begin{array}{l}\text { Duration of stay in } \\
\text { ICU: (days: }\end{array}$ & $2.10 \pm 0.34$ & $4.56 \pm 0.21$ & $3.51 \pm 0.68$ & 71.51 & $<0.001$ \\
\hline $\begin{array}{l}\text { Duration of hospital } \\
\text { stay (days) }\end{array}$ & $2.91 \pm 0.16$ & $9.12 \pm 0.03$ & $6.39 \pm 0.12$ & 620.77 & $<0.001$ \\
\hline $\begin{array}{l}\text { Need for mechanical } \\
\text { ventilation: } \\
\text { Yes } \\
\text { No }\end{array}$ & $\begin{array}{l}98(79.0) \\
26(21.0)\end{array}$ & $\begin{array}{c}33(8.4) \\
242(91.6)\end{array}$ & $\begin{array}{l}131(32.8) \\
268(67.2)\end{array}$ & 174.14 & $<0.001$ \\
\hline
\end{tabular}


Table 2 : Poisoning data of aluminum and zinc phosphide poisoning cases as regards their outcome.

\begin{tabular}{|c|c|c|c|c|c|}
\hline Poisoning data & $\begin{array}{c}\text { Non- } \\
\text { survivors } \\
(\mathrm{n}=124) \\
\text { No }(\%)\end{array}$ & $\begin{array}{c}\text { Survivors } \\
(\mathrm{n}=275) \\
\text { No }(\%)\end{array}$ & $\begin{array}{c}\text { Total } \\
(\mathrm{n}=399) \\
\text { No }(\%)\end{array}$ & $\chi^{2}$ & p-value \\
\hline $\begin{array}{l}\text { Types of poisoning: } \\
\text { Zinc phosphide } \\
\text { Aluminum phosphide }\end{array}$ & $\begin{array}{c}3(2.4) \\
121(97.6)\end{array}$ & $\begin{array}{l}216(78.5) \\
59(21.5)\end{array}$ & $\begin{array}{l}219(54.9) \\
180(45.1)\end{array}$ & 200.02 & $<0.001$ \\
\hline $\begin{array}{l}\text { Manner of poisoning: } \\
\text { Suicidal } \\
\text { accidental }\end{array}$ & $\begin{array}{c}119(95.9) \\
5(4.1)\end{array}$ & $\begin{array}{c}249(90.5) \\
26(9.5)\end{array}$ & $\begin{array}{l}368(92.2) \\
31(7.8)\end{array}$ & 3.51 & 0.061 \\
\hline $\begin{array}{l}\text { Route of intake: } \\
\text { Oral }\end{array}$ & $124(100.0)$ & $275(100.0)$ & $399(100.0)$ & - & - \\
\hline $\begin{array}{l}\text { Formula: } \\
\text { Tablets } \\
\text { Powder }\end{array}$ & $\begin{array}{c}121(97.6) \\
3(2.4)\end{array}$ & $\begin{array}{l}59(21.5) \\
216(78.5)\end{array}$ & $\begin{array}{l}180(45.1) \\
219(54.9)\end{array}$ & 200.02 & $<0.001$ \\
\hline $\begin{array}{l}\text { No of Alp tablets*: }(n=180) \\
<1 \\
\geq 1\end{array}$ & $\begin{array}{c}18(14.5) \\
103(83.1) \\
\end{array}$ & $\begin{array}{c}53(19.3) \\
6(2.2) \\
\end{array}$ & $\begin{array}{c}71(17.8) \\
109(27.3)\end{array}$ & 93.53 & $<0.001$ \\
\hline $\begin{array}{l}\text { No of zinc phosphide } \\
\text { powder packets } * *:(N=219)<0.5 \\
0.5-1\end{array}$ & $\begin{array}{c}0 \\
3(2.5)\end{array}$ & $\begin{array}{l}213(77.4) \\
3(1.1)\end{array}$ & $\begin{array}{c}213(53.4) \\
6(1.5)\end{array}$ & 107.98 & $<0.001$ \\
\hline $\begin{array}{l}\text { Time from intake to } \\
\text { admission (hours): } \\
1-2 \\
>2\end{array}$ & $\begin{array}{c}12(9.7) \\
112(90.3)\end{array}$ & $\begin{array}{l}226(82.2) \\
49(17.8)\end{array}$ & $\begin{array}{l}238(59.6) \\
161(40.4)\end{array}$ & 186.66 & $<0.001$ \\
\hline $\begin{array}{l}\text { Referred from other } \\
\text { medical care unit: } \\
\text { Yes } \\
\text { No }\end{array}$ & $\begin{array}{l}63(50.8) \\
61(49.2) \\
\end{array}$ & $\begin{array}{c}89(32.3) \\
186(67.6)\end{array}$ & $\begin{array}{l}152(38.1) \\
247(61.9) \\
\end{array}$ & 12.33 & $<0.001$ \\
\hline
\end{tabular}

*AlP in 3 grams tablet (57\% AlP; $1.7 \mathrm{~g} /$ tablet)

**: Zinc phosphide in 5 grams powder packet (80\% zinc phosphide; $4 \mathrm{~g} /$ packet) 
Table 3: Clinical manifestations of aluminum and zinc phosphide poisoning cases as regards their outcome.

\begin{tabular}{|c|c|c|c|c|c|}
\hline Clinical manifestations & $\begin{array}{c}\text { Non-survivors } \\
(\mathrm{n}=124) \\
\text { No }(\%)\end{array}$ & $\begin{array}{c}\text { Survivors } \\
(\mathrm{n}=275) \\
\text { No }(\%)\end{array}$ & $\begin{array}{c}\text { Total } \\
(\mathrm{n}=399) \\
\text { No }(\%)\end{array}$ & $\chi^{2}$ & p-value \\
\hline $\begin{array}{l}\text { Level of consciousness: } \\
\text { Conscious } \\
\text { Drowsy or confused }\end{array}$ & $\begin{array}{l}52(41.9) \\
72(58.1)\end{array}$ & $\begin{array}{c}217(78.9) \\
58(21.1)\end{array}$ & $\begin{array}{l}269(67.4) \\
130(32.6)\end{array}$ & 53.19 & $<0.001$ \\
\hline $\begin{array}{l}\text { Temperature: } \\
\text { Normal } \\
\text { Hypothermia }\end{array}$ & $\begin{array}{c}109(87.9) \\
15(12.1)\end{array}$ & $\begin{array}{c}271(98.5) \\
4(1.5)\end{array}$ & $\begin{array}{c}380(95.2) \\
19(4.8)\end{array}$ & 21.34 & $<0.001$ \\
\hline $\begin{array}{l}\text { Pulse: } \\
\text { Normal } \\
\text { Tachycardia } \\
\text { Bradycardia }\end{array}$ & $\begin{array}{l}10(8.1) \\
46(37.1) \\
68(54.8)\end{array}$ & $\begin{array}{c}210(76.4) \\
60(21.8) \\
5(1.8) \\
\end{array}$ & $\begin{array}{l}220(55.1) \\
106(26.6) \\
73(18.3)\end{array}$ & 211.13 & $<0.001$ \\
\hline $\begin{array}{l}\text { BP: } \\
\text { Normal } \\
\text { Hypotension }\end{array}$ & $\begin{array}{l}27(21.8) \\
97(78.2) \\
\end{array}$ & $\begin{array}{c}216(78.5) \\
59(21.5) \\
\end{array}$ & $\begin{array}{l}243(60.9) \\
156(39.1) \\
\end{array}$ & 115.68 & $<0.001$ \\
\hline $\begin{array}{l}\text { RR: } \\
\text { Normal } \\
\text { Rapid } \\
\end{array}$ & $\begin{array}{l}25(20.2) \\
99(79.8) \\
\end{array}$ & $\begin{array}{c}207(75.3) \\
68(24.7) \\
\end{array}$ & $\begin{array}{l}232(58.1) \\
167(41.9) \\
\end{array}$ & 106.66 & $<0.001$ \\
\hline $\begin{array}{l}\text { Skin colour: } \\
\text { Normal } \\
\text { Cyanosed } \\
\end{array}$ & $\begin{array}{l}63(50.8) \\
61(49.2) \\
\end{array}$ & $\begin{array}{c}272(98.9) \\
3(1.1) \\
\end{array}$ & $\begin{array}{c}335(84.0) \\
64(16.0) \\
\end{array}$ & 146.84 & $<0.001$ \\
\hline $\begin{array}{l}\text { Vomiting: } \\
\text { Present } \\
\text { Absent }\end{array}$ & $\begin{array}{c}118(95.2) \\
6(4.8)\end{array}$ & $\begin{array}{c}208(75.6) \\
67(24.4)\end{array}$ & $\begin{array}{c}326(81.7) \\
73(18.3)\end{array}$ & 21.80 & $<0.001$ \\
\hline $\begin{array}{l}\text { Abdominal pain: } \\
\text { Present } \\
\text { Absent }\end{array}$ & $\begin{array}{l}92(74.2) \\
32(25.8) \\
\end{array}$ & $\begin{array}{c}219(79.6) \\
56(20.4)\end{array}$ & $\begin{array}{c}311(77.9) \\
88(22.1)\end{array}$ & 1.47 & 0.225 \\
\hline $\begin{array}{l}\text { Poisoning severity score: } \\
\text { None } \\
\text { Mild } \\
\text { Moderate } \\
\text { Severe }\end{array}$ & $\begin{array}{c}0 \\
0 \\
10(8.1) \\
114(91.9)\end{array}$ & $\begin{array}{c}56(20.4) \\
213(77.4) \\
6(2.2) \\
0\end{array}$ & $\begin{array}{l}56(14.0) \\
213(53.4) \\
16(4.0) \\
114(28.6)\end{array}$ & 381.49 & $<0.001$ \\
\hline
\end{tabular}


Table 4: Investigations of aluminum and zinc phosphide poisoning cases as regards their outcome.

\begin{tabular}{|c|c|c|c|c|c|}
\hline Investigations & $\begin{array}{c}\text { Non- } \\
\text { survivors } \\
(\mathrm{n}=124) \\
\text { No }(\%)\end{array}$ & $\begin{array}{c}\text { Survivors } \\
(\mathrm{n}=275) \\
\text { No }(\%)\end{array}$ & $\begin{array}{c}\text { Total } \\
(\mathrm{n}=399) \\
\text { No }(\%)\end{array}$ & $\chi^{2}$ & $\begin{array}{c}\mathrm{p}- \\
\text { value }\end{array}$ \\
\hline $\begin{array}{l}\text { Creatinine: } \\
\text { Normal } \\
\text { Elevated }\end{array}$ & $\begin{array}{c}107(86.3) \\
17(13.7)\end{array}$ & $\begin{array}{c}270(98.2) \\
5(1.8)\end{array}$ & $\begin{array}{c}377(94.5) \\
22(5.5)\end{array}$ & 23.20 & $<0.001$ \\
\hline $\begin{array}{l}\text { K level: } \\
\text { Normal } \\
\text { Hypokalemia } \\
\text { hyperkalemia }\end{array}$ & $\begin{array}{c}94(75.8) \\
24(19.4) \\
6(4.8) \\
\end{array}$ & $\begin{array}{l}251(91.3) \\
21(7.6) \\
3(1.1) \\
\end{array}$ & $\begin{array}{c}345(86.5) \\
45(11.3) \\
9(2.2) \\
\end{array}$ & 11.73 & 0.001 \\
\hline $\begin{array}{l}\text { Magnesium level: } \\
\text { Normal } \\
\text { Hypomagnesaemia }\end{array}$ & $\begin{array}{c}113(91.1) \\
11(8.9)\end{array}$ & $\begin{array}{c}267(97.1) \\
8(2.9)\end{array}$ & $\begin{array}{c}380(95.2) \\
19(4.8)\end{array}$ & 6.70 & 0.010 \\
\hline $\begin{array}{l}\text { pH: } \\
\text { Normal } \\
\text { Acidosis }\end{array}$ & $\begin{array}{c}14(11.3) \\
110(88.7)\end{array}$ & $\begin{array}{c}253(92.0) \\
22(8.0)\end{array}$ & $\begin{array}{l}267(66.9) \\
132(33.1)\end{array}$ & 251.47 & $<0.001$ \\
\hline $\begin{array}{l}\mathrm{NaHCO}_{3:} \\
\text { Normal } \\
\text { Decreased }\end{array}$ & $\begin{array}{c}19(15.3) \\
105(84.7)\end{array}$ & $\begin{array}{c}215(78.2) \\
60(21.8)\end{array}$ & $\begin{array}{l}234(58.6) \\
165(41.4)\end{array}$ & 139.24 & $<0.001$ \\
\hline $\begin{array}{l}\mathbf{P a O}_{2}: \\
\text { Normal } \\
\text { Hypoxia }\end{array}$ & $\begin{array}{c}10(8.1) \\
114(91.9)\end{array}$ & $\begin{array}{c}272(98.9) \\
3(1.1)\end{array}$ & $\begin{array}{l}282(70.7) \\
117(39.3)\end{array}$ & 340.02 & $<0.001$ \\
\hline $\begin{array}{l}\mathrm{PaCO}_{2}: \\
\text { Normal } \\
\text { Increased }\end{array}$ & $\begin{array}{c}102(82.3) \\
22(17.7)\end{array}$ & $\begin{array}{c}272(98.9) \\
3(1.1)\end{array}$ & $\begin{array}{c}374(93.7) \\
25(6.3)\end{array}$ & 40.35 & $<0.001$ \\
\hline $\begin{array}{l}\text { EF: } \\
\text { Normal (50-70\%) } \\
\text { Mild dysfunction }(40-49 \%) \\
\text { Moderate dysfunction }(30-39 \%) \\
\text { Severe dysfunction }(<30 \%) \\
\end{array}$ & $\begin{array}{c}0 \\
2(1.6) \\
44(35.5) \\
78(62.9) \\
\end{array}$ & $\begin{array}{c}216(78.5) \\
53(19.3) \\
6(2.2) \\
0 \\
\end{array}$ & $\begin{array}{l}216(54.2) \\
55(13.8) \\
50(12.5) \\
78(19.5) \\
\end{array}$ & 365.35 & $<0.001$ \\
\hline $\begin{array}{l}\text { Silver nitrate screening: } \\
\text { Negative } \\
\text { Positive }\end{array}$ & $\begin{array}{c}0 \\
124(100)\end{array}$ & $\begin{array}{c}78(28.4) \\
197(71.6)\end{array}$ & $\begin{array}{c}78(19.5) \\
305(80.5)\end{array}$ & 43.72 & $<0.001$ \\
\hline $\begin{array}{l}\text { Decreased cholinesterase level } \\
\text { Yes } \\
\text { No } \\
\end{array}$ & $\begin{array}{c}6(4.8) \\
118(95.2) \\
\end{array}$ & $\begin{array}{c}10(3.6) \\
265(96.4)\end{array}$ & $\begin{array}{c}16(4.1) \\
383(95.9) \\
\end{array}$ & 0.32 & 0.571 \\
\hline
\end{tabular}

EF: ejection fraction 
Table 5: Binary logistic regression analysis for relevant predictors of mortality in aluminum and zinc phosphide poisoning cases.

\begin{tabular}{|l|l|l|l|l|}
\hline Predictors & P- & OR & \multicolumn{2}{l|}{ 95\% C.I. } \\
\cline { 3 - 6 } & value & & $\begin{array}{l}\text { Lower } \\
\text { limit }\end{array}$ & $\begin{array}{l}\text { Upper } \\
\text { limit }\end{array}$ \\
\hline EF: & & & & \\
Normal & & Reference & & \\
Mild dysfunction & 0.180 & 0.36 & 0.081 & 1.603 \\
Moderate dysfunction & 0.006 & 2.16 & 1.032 & 4.668 \\
Severe dysfunction & $<0.001$ & 9.35 & 1.156 & 5.264 \\
\hline Types (Aluminum phosphide) & $<0.001$ & 7.65 & 1.65 & 3.697 \\
\hline$\geq 1.7$ gram of AlP & $<0.001$ & 7.30 & 1.317 & 5.293 \\
\hline Need for mechanical ventilation & $<0.001$ & 5.36 & 1.695 & 3.689 \\
\hline Time delay (> 2 hours) & 0.021 & 2.65 & 0.023 & 0.392 \\
\hline pH (acidosis) & 0.005 & 1.86 & 1.368 & 1.871 \\
\hline Hypotension & 0.014 & 1.46 & 1.080 & 1.977 \\
\hline
\end{tabular}

OR, odds ratio; $\mathrm{CI}$, confidence interval

Duration of hospital stay, duration of stay in ICU, poisoning severity score, $\mathrm{O}_{2}$ levels

were included in regression model but weren't significant.

\section{DISCUSSION}

Aluminum and zinc phosphide are extremely toxic compounds and resulted in a high case fatality rate (124/399 cases; $31.1 \%)$ in the present study. This result was in agreement with a retrospective 7 years study from 2000-2007 in Tehran that showed an overall mortality rate of $31 \%$ due to aluminum phosphide ( Shadnia et al., 2009).

The case fatality rate from aluminum phosphide alone was about 67\% (121/180 cases) and constituted the majority of deaths among all phosphide deaths (121/124 cases; 97.6\%). Moreover, acute toxicity from aluminum and zinc phosphide constituted the first leading cause of death (124/153 cases; $81 \%$ of total deaths) among all deaths from poisoning cases admitted to Menoufia University Poison and Dependence Control Center (MPCC), Egypt during the period of the present study. These results reflect the bad prognosis and high fatality of phosphide poisoning that need urgent intervention and implementation of policies to ban its use and accessibility. Also, this study revealed that, left ventricular dysfunction, aluminum phosphide poisoning with dose $\geq 1.7$ gram, hypotension, blood $\mathrm{pH}$, and time delay from consumption of poisoning to treatment were found to be the most important predictors of death in phosphide poisoning cases.

Suicide was the most common manner of phosphide poisoning as these compounds are easy to access and cheap with rapid action and high fatality. This may direct persons to use aluminum and zinc phosphide for suicide. Similar findings were reported by previous study from Iran (Mehrpour et al., 2008) and India (Louriz et al., 2009). Many factors such as family conflicts, drug and alcohol abuse, emotional distress, depression, medical diseases, social isolation, and financial and work-related problems may contribute to suicidal acts (Konradsen et al., 2003) 
The most common age for phosphide poisoning in the present study was less than 18 years followed by those aged from 18-30 years as these ages are more susceptible to social and psychological stress so become more vulnerable to commit suicide (Alinejad et al., 2017). They also may have conflict with other family members or friends and emotional problems (Chang et al., 2011). Also, Mathai and Bhanu (2010) found that the majority of acute aluminum phosphide poisoning patients were young and in the age group from 21 to 40 years.

Aluminum and zinc phosphide poisoning was more common in females than males in our study with a ratio of about 2:1 as females attempt suicide greater than in males due to females restricted freedom, harmful traditional practices and frustration of females in Egyptian community from other family members. These results were in agreement with another Egyptian study (EI Naggar et al., 2011). On the contrary, Darbari et al., 2008 and Saha et al., 2015 reported that aluminum phosphide poisoning more common in males than females. This disagreement may be due different life style and different traditions between different countries.

More than two thirds of cases were single $(67.2 \%)$ as married persons are more emotionally and psychologically stable. Also, about half of the cases were students (48.9\%), this could be explained as students are susceptible to more emotional stress and conflict with others and hence more tendency to commit suicide. Housewives, farmers and workers were also common as these slices are more involved in grain storing and so phosphides toxicity.

Most of cases belonged to the rural area. Menoufia is an agriculture governorate, also grain storing is more in rural than urban areas. This is in accordance with previous studies Kapoor et al. (2006) who found that $65.1 \%$ of aluminum phosphide poisoning cases were from rural areas.

The first presentation of phosphide poisoning were normal in most patients especially who transferred to the hospital within the first hours of poison ingestion. Systemic symptoms developed within the first 24 hours as vomiting and abdominal pain, hypotension, tachycardia, tachypnea, cyanosis and changes in the levels of consciousness. Finally, the patients proceed to multi-organ failure and death within the first 24 to 48 hours after poisoning. Similar findings were also reported in many previous studies from different countries (Anger et al., 2000; Nocera et al., 2000; Navabi et al., 2018).

The fatality from phosphide poisoning showed a statistically significant relationship with many variables including aluminum phosphide in a dose $\geq 1.7$ gram (one tablet or more). Aluminum phosphide in Egypt is available in a 3 grams tablet form (containing $57 \%$ aluminum phosphide active ingredient equivalent to $1.7 \mathrm{~g} /$ tablet) while zinc phosphide is formulated as a 5 grams powder packet (containing $80 \%$ zinc phosphide active ingredient; $4 \mathrm{~g} /$ packet) (National Pesticide Information Center, 2011). This was supported by Hajouji et al. (2006) who stated that death from aluminum phosphide is common if more than $1.5 \mathrm{~g}$ is ingested.

Zinc phosphide is less dangerous than aluminum phosphide because it does not produce as much phosphine gas. This is due to zinc phosphide requires acidic conditions for appreciable hydrolysis and subsequent formation of phosphine, whereas aluminum phosphide will hydrolyze to form phosphine in neutral $\mathrm{pH}$ (Knight, 2006).

Left ventricular dysfunction was still the most predictor factor for mortality in phosphide poisoning in this study after multivariate logistic regression analysis; severe and moderate left ventricular dysfunction was the most predicting factor of death followed by the need for mechanical ventilation, aluminum 
phosphide in a dose $\geq 1.7$ gram, hypotension and acidosis as cardiogenic shock secondary to toxic myocarditis induced by phosphine release was reported as the most common cause of death following aluminum phosphide poisoning in many previous studies (Louriz et al., 2009; Mehrpour et al., 2012; Proudfoot et al., 2009).

Metabolic acidosis, hypokalemia, hyperkalemia, hypomagnesaemia, hypoxemia and elevated serum creatinine, were significantly more prevalent among the non-survivors than survivors. Hypokalemia might be contributed to vomiting that presented in $95.2 \%$ of nonsurvivors. These findings were in agreement with Gurjar et al. (2011) who reported that manifestations like refractory shock, metabolic acidosis, severe hypoxia and electrolyte imbalance were observed in cases of AlP poisoning and they are associated with poor prognosis. This could be explained by the release of phosphine gas which inhibits mitochondrial cytochrome oxidase and cellular oxygen utilization and so causing cellular hypoxia and metabolic acidosis (Wang et al., 2007).

It has been found that organs with a higher need for oxygen as heart, lung, kidney, and liver are more sensitive to damage by phosphine gas as phosphine also produces oxygen free radicals in body tissues. In AlP poisoning patients may remain conscious till the late stage but can have manifestations like headache, dizziness, confusion, convulsion and coma due to affection of aerobic metabolism of cerebral cells by inhibition of mitochondrial cytochrome oxidase enzyme. Blockage of oxidative phosphorylation and poor tissue perfusion lead to accumulation of lactic acid and metabolic acidosis (Goel \& Aggarwal, 2007).

Moghadamnia (2012) and Chugh, et al. (1990) reported that decreased plasma magnesium and decreased or increased potassium levels may occur and presence of metabolic acidosis indicates moderate to severe AlP overdose.

The present study showed that $80.5 \%$ of the cases were positive silver nitrate screening test. It is a chemical qualitative color test that was done to detect phosphine (PH3) in stomach contents. Similarly Soltaninejad et al. (2009) indicated that $\mathrm{PH} 3$ detection with silver nitrate test is the commonest and most valuable in clinical and forensic tests that can be done on bio samples such as stomach content and expiratory air and is sensitive enough to detect very low concentrations of $\mathrm{PH} 3$ as $0.05 \mathrm{mg} \mathrm{L}-1(0.05$ ppm).

Positive silver nitrate screening test was more prominent in non-survivors than survivors and this may be due to the fact that non-survivors usually ingest higher amounts of the poison hence more concentration of $\mathrm{PH} 3$ in the stomach content.

In this study only $4.1 \%$ showed decreased cholinesterase level (by about $65 \%$ of normal) but with no relevant manifestations of cholinesterase inhibition. Many investigations previously reported that phosphides inhibit cholinesterase activity as Saidi and Shojaie. (2012) who found that plasma cholinesterase levels were inhibited in rat group receiving ALP. Also Hashemi-Domeneh et al. (2016) and Mittra et al. (2001) stated that both ALP and phosphine cause cholinesterase inhibition, but this inhibition is unlikely to be clinically relevant.

In addition, More than 2 hours' time delay to attend medical care was still predictors of death in phosphide poisoning patients on multivariate logistic regression. Similarly, Navabi et al., reported that the elapsed time from consumption to treatment was $1.3 \pm 0.7$ and $2.0 \pm 1.4$ hours for survivors and non- survivors of ALP poisoning, respectively. Therefore, accelerating the transport of poisoned individuals to treatment centers, followed by careful attention to blood pressure and blood $\mathrm{pH}$ at the time of treatment, would 
be expected to reduce the risk of mortality in cases of aluminum phosphide poisoning.

Regarding period of stay in hospital, it was longer in survivors than non-survivors as most non-survivors came to emergency room with very severe cardiogenic shock that caused death in less than 48 hours in the majority of the cases.

A significantly higher percentage of non survivors than survivors were referred cases. this Only one factor of the cause of the previous finding is improper management; other factors may play a role as remoteness of the cases taking long hours to reach medical care or delay due to undetected cases (non- revealing suicidal patients). Also, gastric lavage might have been done by water which enhances release of phosphine gas in the stomach and hence more toxicity

The present study had some limitation as it was conducted in a single center, the findings may not be representative to the general population in Egypt but it can provide useful data for healthcare providers about the pattern of phosphide poisoning and risk factors of mortality from these types of pesticides.

\section{CONCLUSIONS \& RECOMMENDATIONS}

The results of this study showed that mortality can be predicted in patients with phosphide poisoning based on their type whether aluminum or zinc phosphide, formula and dose of poison. In addition to, left ventricular function, blood pressure, blood $\mathrm{pH}$, and time delay from consumption to treatment. These finding necessitate the need for public health policies to restrict the access and use of phosphides in Egypt with the awareness of the public to the high fatality of these poisons and to encourage the healthcare providers to take more effective measures to treat patients with phosphide poisoning.

\section{REFERENCES}

Ahmed, H.A. (2017): Determination of zinc phosphide and related heavy metals in biological samples. A case report. Asian Journal of Chemistry, 29 (7):1220-1224.

Alinejad, S.; Zamani, N; Abdollahi, M. and Mehrpour, O.A. (2017): Narrative Review of Acute Adult Poisoning in Iran. Iran $\mathrm{J}$ Med Sci, 42(4):327-346.

Anger, F.; Paysant, F.; Brousse, F.; Le Normand, I.; Develay, P.; Gaillard, Y.; et al. (2000) : Fatal aluminum phosphide poisoning. J Anal Toxicol, 24:9092.

Available

at:

http://content.onlinejacc.org/article.asp $\mathrm{x}$ ? articleid $=1695825$

Chang, B.; Gitlin, D. and Patel, R. (2011): The depressed patient and suicidal patient in the emergency department. Evidence-based management and treatment strategies. Emergency Medicine practice, 13(9):1-23.

Chaudhry, D. and Rai,A.S. (2014): Nacetyl cysteine in aluminum phosphide poisoning: Myth or hope. Indian J Crit Care Med, 18:646-647.

Chugh, S.N.; Juggal, K.L.; Sharma, A.; Arora, B. and Malhotra, K.C. (1990): Magnesium levels in aluminum phosphide poisoning. $\mathrm{J}$ Assoc Physicians India, 38:32.

Darbari, A.; Tandon, S.; Chaudhary, S.; Bharadwaj, M.; Kumar, A. and Singh, G.P. (2008): Esophageal injuries due to aluminum phosphide tablet poisoning in India. Asian Cardiovascular and Thoracic Annals, 16(4):298-300.

El Naggar, A. M. and El Mahdy, N. M. (2011): Zinc phosphide toxicity with a trial of tranexamic acid in its management. Journal of Advanced Research, 2(2):149-156.

Goel, A. and Aggarwal, P. (2007): Pesticide poisoning. Natl Med J India, 20:182-191.

Gurjar, M.; Baronia, A.K.; Azim, A. and Sharma, K.(2011): Managing 
aluminum phosphide poisonings. J Emerg Trauma Shock, 4(3):378-384.

Hajouji, I.M.; Oualili, L.; Abidi, K.; Abouqal, R.; Kerkeb, $O$. and Zeggwagh, A.A.(2006): Severity factors of aluminum phosphide poisoning (phostoxin) Ann Fr Anesth Reanim, 25:382-385.

Hashemi-Domeneh, B.; Zamani, N.; Hassanian-Moghaddam, H. Rahimi, M.; Shadnia, S., Erfantalab, P.; et al. (2016): A. A review of aluminum phosphide poisoning and a flowchart to treat it. Arh Hig Rada Toksikol, 67:183-193.

Kaplan, L. A.; Tobor, M. W. and Pesce, A.J. (1989): chromatography (Theory, analysis and correlation). $1^{\text {st }}$ ed. Mosby. CO. USA. 73-93.

Kapoor, A.K.; Shina, U.S.; Singh, U.S. and Mehtrotra, R.(2006): An epidemiological study of aluminium phosphide poisoning at Allahabad. Int J Forensic Med Ther, 4:1-7.

Knight, M. W.(2006): Zinc Phosphide. Small Animal Toxicology, 2nd ed.; Peterson, M. E.; Talcott, P. A. Eds.; Elsevier Saunders: Saint Louis, MO, 1101-1118.

Konradsen, F.; Van der Hoek, W.; Cole, D.C.; Hutchinson, G.; Daisley, H.; Singh, S.; et al. (2003): Reducing acute poisoning in developing countries--options for restricting the availability of pesticides. Toxicology, 192(2-3):249-261.

Louriz, M.; Dendane, T.; Abidi, K.; Madani, N.; Abouqal, $R$. and Zeggwagh, A. (2009): Prognostic factors of acute aluminum phosphide poisoning. Indian journal of medical sciences, 63(6):227-234.

Mathai, A. and Bhanu, M.S. (2010): Acute aluminium phosphide poisoning: Can we predict mortality? Indian J Anaesth, 54:302-307.

Mehrpour, O.; Alfred, S.; Shadnia, S.; Keyler, D.; Soltaninejad, K.; Chalaki, N.; et al. (2008): Hyperglycemia in acute aluminum phosphide poisoning as a potential prognostic factor. Human \& experimental toxicology, 27(7):591595.

Mehrpour, O.; Jafarzadeh, M. and Abdollahi, M. A.(2012): systematic review of aluminium phosphide poisoning. Arh Hig Rada Toksikol, 63:61-73.

Mittra, S.; Peshin, S. and Lall, S.(2001): Cholinesterase inhibition by aluminum phosphide poisoning in rats and effects of atropine and pralidoxime chloride. Acta Pharmacol Sin, 22:37-39 .PMID: 11730559

Moghadamnia, A.A.(2012): An update on toxicology of aluminum phosphide .DARU Journal of Pharmaceutical Sciences, 20:25.

National Pesticide Information Center (2011): Zinc phosphide technical fact sheet.npic@ace.orst.edu.

Navabi, S.; Navabi, J.; Aghaei, A.; Shaahmadi, Z. and Heydari, R. (2018): Mortality from aluminum phosphide poisoning in Kermanshah Province, Iran: characteristics and predictive factors. Epidemiol Health, 40:e2018022.

https://doi.org/10.4178/epih.e2018022

Nocera, A.; Levitin, H.W. and Hilton JM.(2000): Dangerous bodies: a case of fatal aluminium phosphide poisoning. Med J Aust,173:133-135.

Persson HE, Sjoberg GK, Haines JA, Pronczuk de GJ. (1998): Poisoning severity score. Grading of acute poisoning .J toxicol Clinical Toxicol, 6 (3):250-255.

Proudfoot, A.T. (2009): Aluminium and zinc phosphide poisoning. Clin Toxicol (Phila) 47:89-100.

Saha, J.K.; Azad, K.A.; Hossain, M.Z.; Amin, M.R.; Ahmed, M.; Ahsan, H.M.; et al. (2015): Aluminium phosphide poisoning cases in a tertiary care hospital. J Dhaka Med Coll, 23:36.

Saidi, H. and Shojaie, S. (2012): Effect of sweet almond oil on survival rate and 
plasma cholinesterase activity of aluminum phosphide-intoxicated rats. Human \& experimental toxicology, 31(5), 518-522.

Shadnia, S.; Rahimi, M.; Pajoumand, A.; Rasouli, M.H. and Abdollahi, M.(2005): Successful treatment of acute aluminium phosphide poisoning: possible benefit of coconut oil. Human \& experimental toxicology, 24(4):2158.

Shadnia, S.; Sasanian, G.; Allami, P.; Hosseini, A.; Ranjbar, A.; AminiShirazi, N.; et al. (2009): A retrospective 7-years study of aluminum phosphide poisoning in Tehran: opportunities for prevention. Human \& experimental toxicology, 28 (4): 209-213.

Shah, V.; Baxi, S, and Vyas, T. (2009): Severe myocardial depression in a patient with aluminium phosphide poisoning: A clinical, electrocardiographical and histopathological correlation. Indian J Crit Care Med, 13:4143

Sogut, O.; Baysal, Z. and Ozdemir, B.(2009): Acute pulmonary edema and cardiac failure due to zinc phosphide ingestion. Journal of Emergency Medicine, 40 (6): e117-e 8.

DOI: 10.1016/j.jemermed.2009.05.039

Soltaninejad, K.; Shadnia, S.; Ziyapour, B. and Brent, J. (2009): Aluminum phosphide intoxication mimicking ischemic heart disease led to unjustified treatment with streptokinase. Clin Toxicol (Phila) 47:908-909. doi: 10.3109/15563650903285657.

Valmas, N.; Zuryn, S. and Ebert, P.R. (2008): Mitochondrial uncouplers act synergistically with the fumigant phosphine to disrupt mitochondrial membrane potential and cause cell death. Toxicology, 252:33-39. 17

Wabar, H. (1966): Kinetic colorimetric method for detection of cholinesterase. Dtsch Med Wschr, 91: 127-129.

Wahab, A.; Rabbani, M.U.; Wahab, S. and Khan, R,A. (2009): Spontaneous self-ignition in a case of acute aluminium phosphide poisoning. Am J Emerg Med, 27:752-756

Wang, W.; Winther, J.R. and Thorpe, C. (2007): Erv2p: characterization of the redox behavior of a yeast sulfhydryl oxidase. Biochemistry, 46:3246-3254.

Yancy, C.W.; Jessup, M. and Bozkurt B. (2013): ACCF/AHA Guideline for the Management of Heart Failure: A Report of the American College of Cardiology Foundation/American Heart Association Task Force on Practice Guidelines. J Am Coll Cardiol, 62(16):e147-e239. 